\title{
Commentaires et Discussions
}

\section{STABILITÉ DE RÉGLAGE D'UN GROUPE HYDROÉLECTRIQUE}

\author{
Réponse à $M$. ALMÉRAs
}

L'importance de la question dont il s'agit et dont dépend le choix des caractéristiques de réglage des groupes hydroélectriques - notamment celui de l'inertie de leurs alternateurs me paraît avoir été heureusement saisie par les milieux intéressés. C'est dans ce sens que je salue la parution dans cette Revue des travaux de M. AlméR.s, Ingénieur, et que je remercie la Rédaction de La Houille Blanche de me permettre de m'exprimer, dans ses colonnes, a leur sujet.

Je me plais à recomnaître toute la valeur des recherchus de M. Alméras et si je formulerai, à leur égard, quelques critiques, c'esl d'une part qu'il a hien voulu ne point ignorer l'ouvage que j'ai publié en 1945 (r), puis d'autre part el surtout pour mettre en lumière, dans l'intérèt général, les résultats communs obtenus, à côté de certaines divergences.

Ces résultats concordants sont en effet un peu dissimulés par la différence des notations employées pour les traduire en langage mathématique et, tout en

(1) Contribution à l'Etude des Régulateurs de Vitesse. - Considérations sur le Problème de la Stabilité. Edition La Concorde, Lausanne.

(2) La seule qui intéresse a la fois exploitants et constructeurs. me gardant de vouloir instaurer en cette matière une terminologie et une notation définitives, je crois qu'il y aurait intérêt à en chercher de plus claires et de plus expressives que celles utilisées par M. Alméras. Mais ceci n'est qu'une question de forme.

M. Alméras lui-même s'y est laiss é prendre, puisqu'il a d'abord annoncé à ses lecteurs des conditions de stabilité « entì̀rement différentes» de celles que j'avais établies. Il l'avait attribué à une erreur grossière gu'il m'avait faussement imputée ; mais comme il a bien voulu, sur ma demande, se rétracter à ce sujet, j'aurais aujourd'hui mauvaise gràce à insister.

Toutefois, puisque cette divergence originelle n'existe pas, pourquoi subsisterait-il des différences de résultats qui ne seraient pas tout simplement explicables du fait des méthodes de calcul suivies par l'un et par l'autre? M. Alméras traite le coup de bélier comme un phénomène « de masse »; je l'ai

$$
\begin{aligned}
\mathcal{C}= & \text { temps caractéristique dé la promptitude du réglage : } \frac{1}{K_{n}} \text { selon } \\
& \text { M. Alméras. } \\
\mathrm{T}= & \text { temps camactéristique de l'inertie spécifique du groupe : } \boldsymbol{\tau} \text { selon } \\
& \text { M. Alméras. } \\
\Theta= & \text { temps earactéristique de la sensibilité, au coup de bélier, du système } \\
& \text { d'alimentation de la turbine : } \Theta \text { selon M. Alméras. } \\
\mathbf{K}= & \text { une constante, sans dimensions. }
\end{aligned}
$$

fait intervenir comme un phénomène «d'ondes» ce qui, ̀̀ tout bién considérer, n'est guère plus compliqué et présente le grand avantage d'une meilleure préci sion. Contrairement à ce que M. Alméras écrit, les deux méthodes ne possèdent pas chacune des limitations : l'une est approchée, mais suffisante dans certains cas, l'autre est exacté et tout à fait générale.

M. Alméras prétend avoir abouti, grâce à sa méthode, à des résultats immédiatement explicites ; ses lecteurs en jugeront eux-mêmes. Mais pourquoi laisser entendre que mes résultats ne seraient point explicites et que leur application nécessiterait des calculs par approximations successives?

J'ai proposé, au contraire, une condition principale de stabilité (2) de la forme suivante, bien déterminée :

$$
\boldsymbol{\tau}^{\top} \mathrm{T}>\mathrm{K}\left(\frac{3}{2} \Theta\right)^{-}
$$

où toutes les grandeurs sont homogénes à des temps: 
Elle s'écrit donc, avec la notation de M. Alméras :

$$
\frac{\tau}{K}>K\left(\frac{3}{2} \Theta\right)^{2}
$$

et l'on reconnaît immédiatement la formule (16) de la deuxième partie de son étude ${ }^{(x)}$ :

$$
\tau>3,74 K_{1,} \Theta=
$$

correspondant à une valeur de la constante $\mathrm{K}$ :

$$
K=1,66
$$

Cette constatation relative à un résultat que je me suis particulièrement altaché à mettre en relief dans mon ourrage, aurait-elle encore échappé à M. Alméras? Est-ce là une de ces conditions « entièrement cifférentes » qu'il avait annoncées?

Je laisse ici intentionnellement de coté le cas du réglage tachymétrique avec asservissement permanent, bien qu'il me serait facile de montrer que la condition publiée à cet égard par M. Alméras, dans la première partie de son étude, ne diffère en principe pas de celle que javais précédemment établie pour ce même cas. Ce système de réglage n'est, pour ainsi dire, jamais employé seul, dans le domaine des turbines hydrauligues.

Restent Ie réglage tachymétrique avec asservissement temporaire et le réglage accélérotachymétrique. Je les ai assimiiés l'un à l'autre, au point de vue de la recherche de la stabilite el je regrette de voir M. Alméras remarquer que j’ai abordé l'étude de l'asservissement temporaire en tenant pour négligeable un terme qui peut,

(1) Voir La Houille Blanche, $\mathrm{n}^{\circ} 2$, mars 1946, p. 137.

(2) Voir Considérations sur le Problème de la Stabilité, chap. VII, par. 48.

(3) Voir op cit., chap. VII, par. 48. en pratique, ne pas toujours l'être. M. Alméras n'ignore en effet pas que l'approximation que je me suis permis est, en réalité, favorable au système dont i] se déclare partisan, car le mieux que l'on puisse dire d'un asservissement temporaire est qu'il se comporte comme un accélérométre, en vue de la stabilisation du réglage.

Désirant mettre l'accent sur ce qui rapproche nos résultats et non sur ce qui les sépare, je n'insisterai pas sur ce point, pas plus que sur la facon dont M. Alméras traite de l'asservissement temporaire; ses lecteur's en jugeront. Je m'abstiendrai également de relever les arguments que M. Alméras invoque en faveur du réglage tachymétrique avec asservissement temporaire, du point de vue des petites variations de charge. Je reste d'avis contraire au sien, mais pense que nos lecteurs pourront se former eux-mèmes une opinion en recourant à nos sources et à l'expérience.

Je répète donc avoir proposé la condition de stabilité précitée pour les deux systemes de r'églage à la fois, en le motivant, et avoir, par mes abaques, fait le calcul de sa constante $K$ pour différentes valeurs de la chute, en admettant pour le décrément Iogarithmique des oscillations de réglage la valeur de 1,38 (rapport de deux amplitudes successives :

$$
e^{1,3 n}=4 \text { (environ) }
$$

Si l'on reprend ce calcul, en se plaçant dans l'hypothèse simplificatrice du coup de bélier « en masse », voici les résultats auxquels on aboutit :

$\varphi_{\prime \prime}=$ déphasage entre l'oscillation de l'écart de réglage et l'oscillation de l'écart de pression,

$r \quad=$ coefficient caractérisant l'effet de la composante de l'oscillation de pression en opposition de phase avec l'oscillation de l'écart de réglage,

$s \quad=$ coeffeient caractérisant l'effet de la composante de l'oscillation de pression en quadrature avec l'oseillation de léeart de réglage,

$X \quad=$ rapport de la période $T$ " de l'oscillation de réglage à la période $\mathrm{T}$ du coup de bélier d'onde dans le systeme d'alimentation de la turbine,

$\rho=$ caracteristique, selon Allievi, du systeme d'alimentation de la turbine.

$$
\begin{aligned}
& \text { lg } \varphi_{\prime \prime \prime}=\frac{T^{\prime \prime}}{\pi \Theta} \text { on } \quad \text { lg } \varphi_{\prime \prime \prime}=\frac{2}{\pi} \frac{\mathrm{x}}{\rho} \\
& r=\cos ^{2 \cdot} \varphi_{m} \text { el } s=\sin ^{2} \varphi_{\prime \prime}
\end{aligned}
$$

d'où :

$$
r+s=1 \quad \frac{s}{r}=t g^{2} \varphi_{m}=\left(\begin{array}{c}
2 \\
\pi
\end{array}\right)^{2}
$$

En combinant ces équations avec celle que j’ai établie (2) ::

$$
\frac{x}{\rho}=2,5 \frac{s}{1-3 r}
$$

on trouve pour la valeur de la constante $K=\frac{s^{2}}{1-3 r} \quad\left(3^{\prime}\right)$ :

$$
\mathrm{K}=1,30 \text { environ }
$$

chiffre indépendant de la chute. 
Or, dans l'hypothèse du coup de bélier d'onde, où la chute intervient, voici les valeurs de

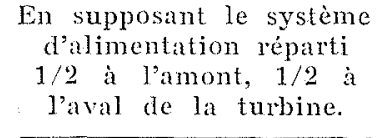

$\mathrm{H}$ (envilous) $=10$

$\mathrm{P}=10$
$\mathrm{I}=1,08$

En comparant les chiffres de ce tablean, avec celui de 1,66 correspondant à la formule (16) de M. Alméras et celui de 1,30 que j'ai moi-même calculé dans l'hypothèse du coup de bélier en masse, on se rend immédiatement compte du degré d'approximation de la dite hypothèse, des cas ou elle est admissible et de ceux où elle ne l'est pas.

Finalement, je constate que la condition principale de stabilité que j'ai établie est retrouvée dans la deuxième partie de l'étude de $M$. Alméras, sans autre remarque de sa part, qu'en vue de son application, M. Alméras n"a apporté aucune facilité nouvelle et que les écarts relevés dans les valeurs de la constante $K$ proviennent des hypothèses admises.

Ceci dit, je me réjouis sincèrement de cette concordance; mais potrquoi s'attacher dès lors à l'hypothèse simplificatrice du coup de bélier en masse qui conduit à une valeur de la constante $K$ indépendante de la chute, alors qu'il est plus juste et pas plus compliqué de faire varier la valeur de cette constante en fonction de la chute?

En outre, il s'agit là d'un résultat théorique, ne tenant pas compte de toutes les contingences de la pratique. J'ai déjà insisté sur le fait qu'il devait donc être soumis à l'épreuve de l'expérience. J'ai publié les résultats de quelques essais effectués. dans ce but par mes soins et je serais particulièrement heureux que d'autres en entreprennent la constante $\mathrm{K}$ que j'ai proposées et motivées :

En supposant le système d'alimentation

ntierement situé à l'amont de la turbine.

$\begin{array}{ccccc}50 & 100 & 500 & 1.000 \mathrm{~m} \\ 2,5 & 1,5 & 0,5 & 0,3 & \\ 1,36 & 1,41 & 1,84 & 2,76\end{array}$

de leur côté, communiquent leurs résultats et contribuent ainsi à faire la lumière sur ce problème dont l'importance et l'intérêt méritent l'attention des exploitants, comme celle des constructeurs.

\section{$* *$}

La première partie de cette rote était rédigée lorsque parut, dans le $n^{\circ} 3$ de cette Revue, le troisième article de $\mathrm{M}$. Alméras comportant son examen de la stabilité de réglage de plusieurs groupes couplés en parallèie, puis les conclusions générales de l'ensemble de son étude. C'est avec un très réel plaisir que j'ai constaté tout le chemin parcouru par M. Alméras, depuis qu'il commença ses publication. sur le sujet que j’ai traité dans İouvrage déjà cité.

En ce qui concerne la stabilité de réglage de plusieurs groupes couplés en parallèle, M. Alméras ecrit lui-même que ses résultat: sont bien exactement ceux que j'avais exposés et l'on pourrait presqu'en dire autant, à quelques détails près, de ses conclusions générales.

M. Alméras a d'ailleurs anne, au mois de juillet à Grenoble une conférence, dont il a bien voulu me communiquer le texte. II $\mathrm{y}$ a reconnu que quel que soit celui des deux systèmes employés - tachymétrique avec asservissement temporaire ou accélérotachymétrique - la recherche de la stabilité procédait des mêmes moyens. C'est bien là un point que je m’étais efforcé, arec insistance, de mettre en relief; mais alors pourquoi ne pas parlér le même langage à propos des deux systèmes, plutôt que d'introduire des notions différentes pour l'un et pour lautre?

Je sais bien que pour en venir, ainsi que je l'ai fait, à considérer le réglage tachymétrique avec asservissement temporaire, comme un réglage accéléro-tachymétrique, il faut résolument se détacher de la conception classique, selon laquelle un asservissement temporaire pourrait être traité comme un asservissement permanent qui disparait avec le temps. Mais pourquoi ne pas faire ce pas en avant puisque, comme je l'ai démontré, cette façon de voir est parfaitement correcte, plus fructueuse en vue de l'expression des résultats et qu'elle permet d'unifier le langage ?

En outre, du point de vue des exploitants, peu leur importe qu'il faille réaliser tel degré efficace ou réel d'un asservissement temporaire, telle valeur de sa rigidité, ou tel rapport entre les rapidités de réponse tachymétrique et accéléro-métrique. Une seule notion me paraît leur être vraiment utile : celle de la promptitude de réglage $d u$ groupe considéré. Cette promptitude définit la vitesse de réglage (en \% de la puissance du groupe, par seconde) dont le groupe est capable, lorsque son régulateur est sollicité par tel écart de fréquence. La dite vitesse de réglage se concoit aussi bien dans le cas du système accéléro - tachymétrique, que dans celui du système tachymétrique avec asservissement temporaire, étant observé que dans le premier elle s'établit immédiatement dès l'apparition du moindre écart (I) et dans le second avec un certain retard, d'où résulte à mes yeux un des avantages évidents du réglage

(1) On peut mème dire abant cette apparition grace au jeu de l'accéléromètre. 
accéléro-tachymétrique. Cette vitesse de réglage peut s'exprimer en kw par sec., elle est mesurable par essais sur le groupe et la promptitude se présente donc comme une grandeur physique bien déterminée, aisément saisissable par l'esprit.

Finalement, Ia préoccupation des exploitants doit être la suivante : Etant donné la sensibilité du système d'alimentation du groupe au phénomène du coup de bélier et l'inertie spécifique de ses masses tournantes, quelle est la promptitude de réglage, compatible avec la stabilité, sur laquelle on peut compter, en d'autres termes quel est Je nombre de $\mathrm{kw}$ par sec. dont le groupe pourra modifier la puissance qu'il produit, lorsque son régulateur sera sollicité par tel écart de fréquence? Puis se pose la question connexe, sur laquelle M. Alméras revient avec raison : Eu égard à l'importance du réseau, à la nature de sa consommation, voire aux conditions de service qui changent selon les heures de la journée, quel est le nombre de kw par sec., dont la consommation peut varier et auquel le ou les groupes réglants doivent satisfaire, en maintenant la fréquence entre telles limites. La comparaison des deux réponses permei de juger de l'inertie spécifique dont les groupes doivent être dotés.

Pour terminer, je noterai encore la confirmation que M. Alméras apporte à l'avis que j'ai expressément formulé : D'une façon générale, le fonctionnement en parallèle de plusieurs groupes n'est pas en lui-même directement générateur de stabilité ; il l'est seulement indirectement :

a) parce que l'importance des variations de charge, auxquelles on peut s'attendre, ne croît pas comme la puissance totale mise en jeu. En valeur relative, cette importance diminue, quand la puissance totale augmente et des résultats expérimentaux seraient, à cet égard, les bienvenus ;

b) parce que la promptitude de réglage de l'ensemble des groupes réglants peut, en conséquence, être réduite, ce qui sera Ie plus facilement obtenu en annulant la promptitude de réglage de certains d'entre-eux, c'est-à-dire en les faisant fonctionner à puissance constante, par le jeu du limiteur d'ouver. ture de leurs régulateurs. On peut dire que ces groupes, qui n'interviendront plus dans le réglage, feront alors cadeau de leur inertie mécanique aux groupes réglants et amélioreront ainsi leurs conditions de stabilité ;

c) parce que certains appareils de consommation sont dotés d'une inertie mécanique et contribuent par là à augmenter l'inertie spécifique de l'ensemble, si du moins la vitesse de ces appareils (moteurs) varie comme celle des groupes générateurs. Cette influence n'est pratiquement pas très grande ;

d) parce qu'enfin - et je voudrais encore insister ici beaucoup plus que $M$. Alméras ne l'a fait - la pente de la loi du couple résistant, en fonction de la fréquence, d'un alterna- teur branché sur le réseau esí favorablement plus élevée (en valeur algébrique) que celle de la loi du couple moteur d'une turbine. Même avec un régulateur de tension, la puissance débitée par un alternateur sur le réseau n'est pas indépendante de la fréquence. Sur ce point également, des résultats expérimentaux seraient les bienvenus, car l'influence dont il s'agit est certaine et sensible.

Cette raison $d$ ) me parait beaucoup plus importante à soumettre à l'examen des électriciens, que celle de l'élasticité du couplage des machines synchrones (oscillation des rotors d'alternateurs dans le champ tournant). Cette élasticité donne lieu à des phénomènes de période (de l'ordre de la seconde) biên plus laible que celle des mouvements de pompage résultant d'une instabilité mécanique (hydraulique). Ils n'ont pratiquement pas à intervenir dans l'examen de cette question, sauf cas exceptionnels de lignes d'interconnexion surchargées au roisinage de leur limite de stabilité électrique.

$\mathrm{Si}$ le fonctionnement en parallèle sur un grand réseau apporte donc indirectement des éléments favorables à la stabilité, il reste à rechercher dans quelle proportion ceci est quantitativement le cas. Le problème de la stabilité de réglage et du PD2 dont les groupes générateurs doivent être dotés, conserve toute son importance, surtout pour des groupes puissants auxquels il doit être fait appel pour participer au réglage du réseau.

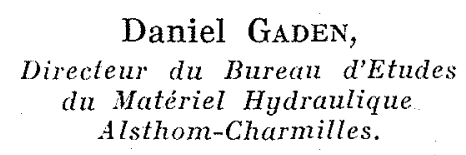

